\title{
Concentration of VFA in the intestinal material and of VFA + ketone bodies in the veins of the large intestine in domestic rabbits
}

\author{
R. BONNAFOUS et P. RAYNAUD \\ Institut de Physiologie, \\ 2, rue François-Magendie, \\ 3 I 400 Toulouse (France)
}

This study was achieved on 2 series of rabbits during the excretion of hard faeces and caecotrophes. Whatever the type of excretion the production and absorption of VFA seemed to take place in the caecum and the proximal colon only. Among the 3 VFA studied (acetic, propionic, butyric), butyric acid seemed to be more easily absorbed.

This could be explained by the conversion of butyrate into ketone bodies which, by shifting its concentration gradient in favour of the intracellular mediun, might activate its diffusion. Contrary to VFA, the production of plasma ketone bodies did not seem to depend on the butyric acid concentration of the material. Moreover, the good concordance beetween the percentage of the $3 \mathrm{VFA}$ in the material and in the plasma suggested a possible absorption of VFA.

\section{Relationship between feed intake and faecal excretion in the doe rabbit just before and after parturition}

\author{
F. LEBAS, J. P. LAPLACE $\left(^{*}\right)$ et Marie-Agnès OGER(*) \\ Laboratoive de Recherches sur l'Élevage du Lapin, \\ Centre de Toulouse, I.N.R.A., \\ 3132o Castanet-Tolosan (France) \\ (*) Laboratoive de Physiologie de la Nutrition, \\ Centre national de Recherches zootechniques I.N.R.A. \\ 78350 Jouy-en-Josas (France)
}

The feed intake and faecal excretion (hard pellets) were observed in ${ }_{5}$ Californian doe rabbits 4 days before and 4 days after parturition.

The minimum ingestion of dry matter ( $40 \mathrm{~g}$ a day) and water was observed on the day just before parturition.

On the contrary, the minimum faecal excretion was observed on the very day of parturition. On the whole a good relationship between dry matter intake and excretion was noticed like in the young growing rabbit. However, these observations suggest a slowing down of the digestive transit rate the days before parturition. Parallel to that, the water absorption might be disturbed the day before parturition. 